\title{
GLAD!
}

Revue sur le langage, le genre, les sexualités

$07 \mid 2019$

Varia

\section{straight nose, queer mouth}

straight nose, queer mouth

\section{Élodie Grethen}

\section{(2) OpenEdition}

Journals

Édition électronique

URL : http://journals.openedition.org/glad/1737

DOI : $10.4000 /$ glad. 1737

ISSN : 2551-0819

\section{Éditeur}

Association GSL

\section{Référence électronique}

Élodie Grethen, « straight nose, queer mouth », GLAD! [En ligne], 07 | 2019, mis en ligne le 05 décembre 2019, consulté le 17 décembre 2020. URL : http://journals.openedition.org/glad/1737 ; DOI : https:// doi.org/10.4000/glad.1737

Ce document a été généré automatiquement le 17 décembre 2020.

\section{(c) (i) (9)}

La revue GLAD! est mise à disposition selon les termes de la Licence Creative Commons Attribution Pas d'Utilisation Commerciale - Pas de Modification 4.0 International. 


\section{straight nose, queer mouth}

straight nose, queer mouth

Élodie Grethen

Image 1

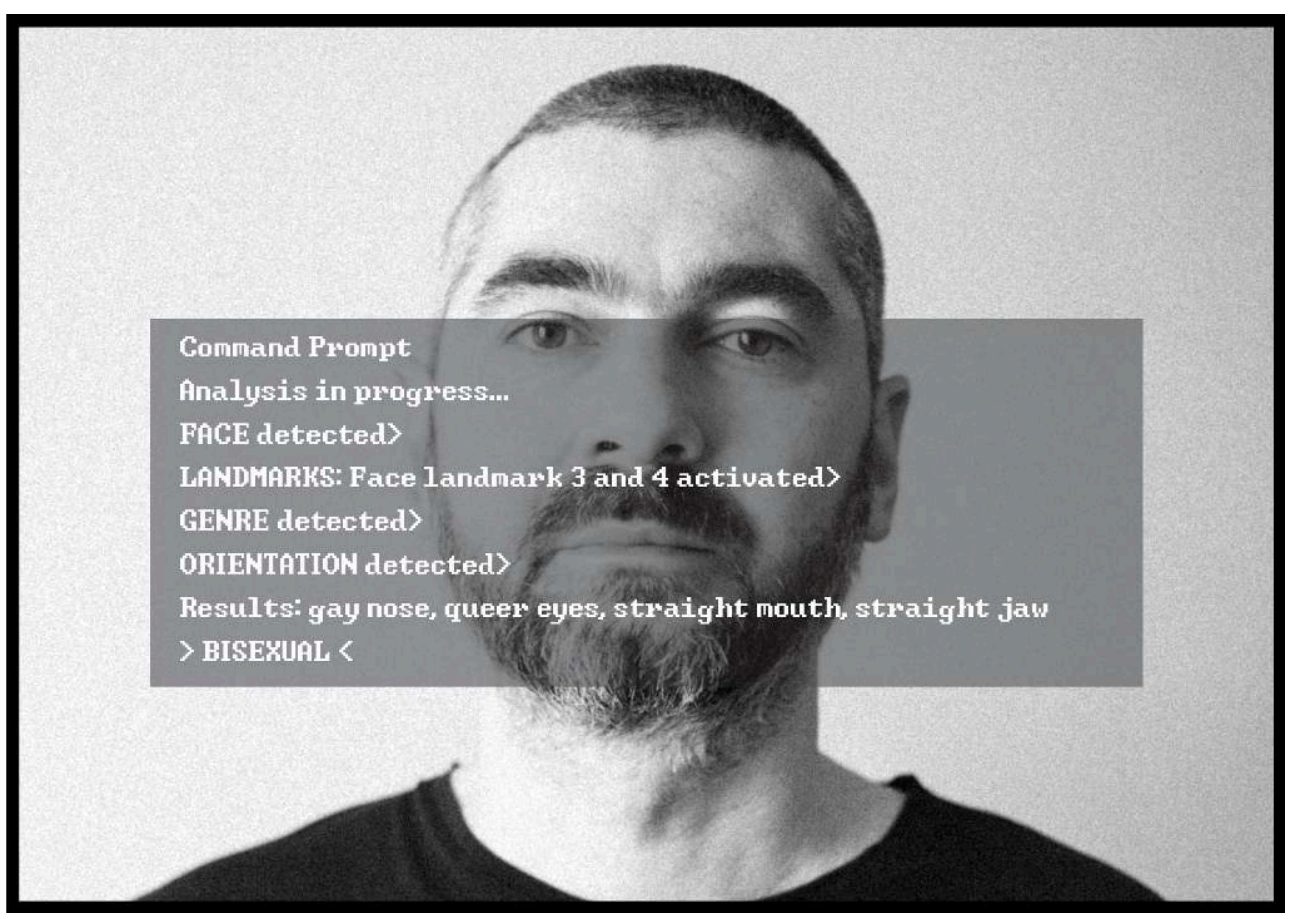




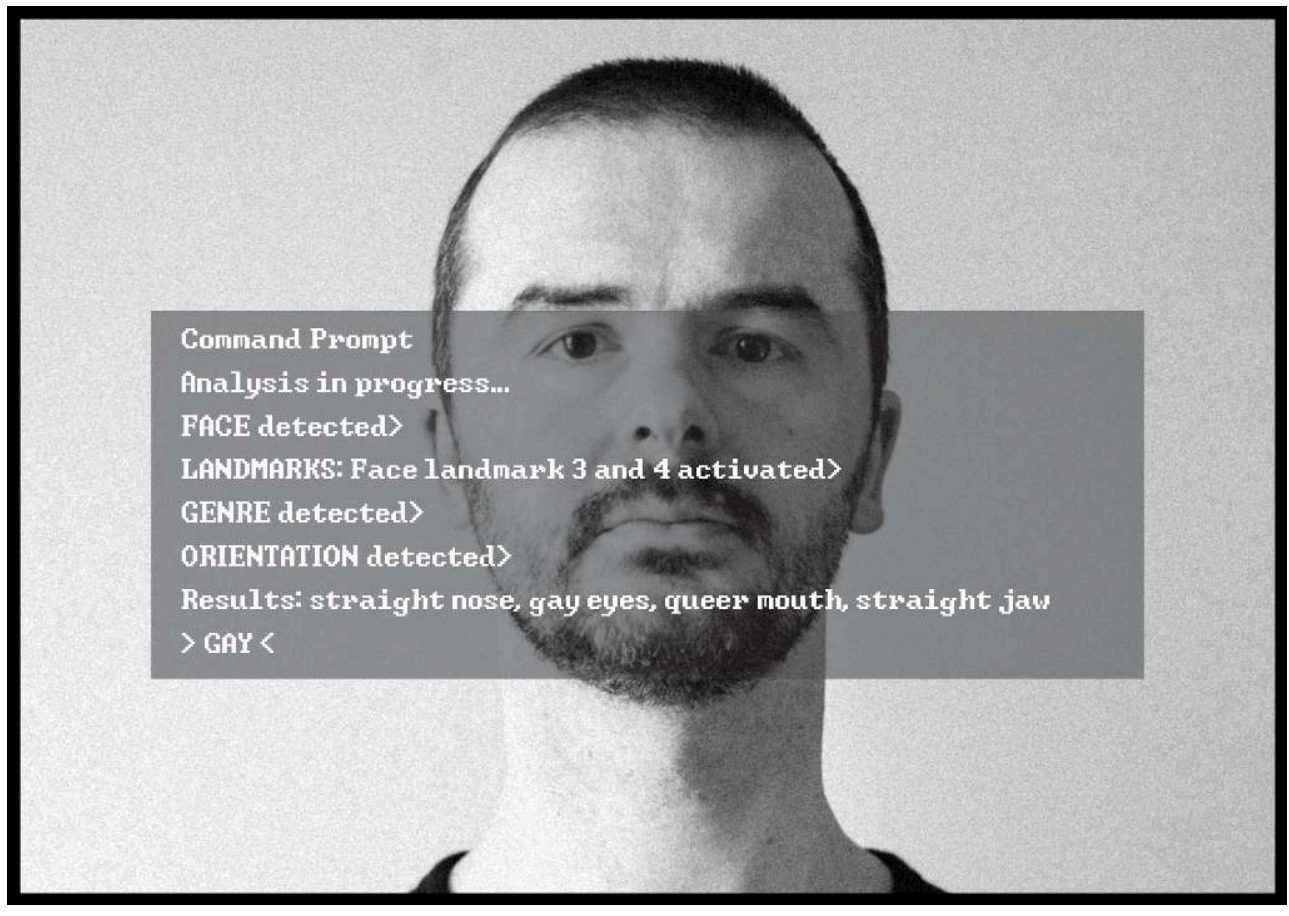

1 Fin 2017, Wang et Kosinski publiaient une étude sur l'orientation sexuelle, qui fit polémique dans les milieux universitaires et sur les réseaux sociaux. En utilisant un réseau neuronal permettant d'extraire les caractéristiques de plus de 35000 images de profil sur un site de rencontres américain, ils ont formé une intelligence artificielle de reconnaissance faciale à identifier et classer l'orientation sexuelle de leurs sujets. L'algorithme pourrait, grâce à l'analyse de ces photos, reconnaître avec une précision allant jusqu'à $91 \%$ si une personne est hétérosexuelle ou homosexuelle.

2 L'étude aurait révélé que «les homosexuels et les lesbiennes tendent à avoir des morphologies faciales, des expressions et des apparences faciales atypiques pour leur genre ». Les hommes homosexuels auraient un menton plus étroit, un front plus haut, un nez plus long et une pilosité faciale moindre que les hommes hétérosexuels, tandis que les lesbiennes auraient un menton plus large et un front plus petit que les femmes hétérosexuelles.

3 Sur la base de ces résultats, Wang et Kosinski affirment que « les visages contiennent bien plus d'informations sur l'orientation sexuelle que ce que le cerveau humain peut percevoir et interpréter », ce qui serait "en adéquation avec la théorie des hormones prénatales sur l'orientation sexuelle ». Ce qui signifie que, en raison de l'exposition à certaines hormones, l'orientation sexuelle est définie avant la naissance : être homo- ou hétérosexuelle n'est pas un choix et se reflèterait donc dans les traits physiques.

4 Bien que le projet photographique "straight nose, queer mouth » ne se concentre pas sur les implications éthiques de cette étude, les dangers d'un tel outil doivent être mentionnés. Alors que les auteurs soutiennent que la technologie de reconnaissance faciale permettant ce type d'étude existe déjà et ne devrait pas être ignorée en raison d'appréhension morale ou idéologique, les organisations LGBTQIA ont manifesté leur inquiétude face à cette étude et ses conclusions. Ils craignent que cette intelligence artificielle puisse être utilisée pour discriminer les personnes homosexuelle's et queer, particulièrement dans les pays où des régimes brutaux sont en place. Le classificateur 
de reconnaissance faciale pourrait également être utilisé par les mouvements antiLGBTQIA pour violer la vie privée des personnes en utilisant des images de profil provenant de médias sociaux ou de données publiques, par exemple par le biais de caméras de surveillance.

5 De plus, l'étude présente une vision très binaire et hétéronormative de la sexualité, les scientifiques et l'algorithme ne considérant aucune autre orientation sexuelle que l'hétérosexualité et l'homosexualité. En excluant de leur recherche les personnes bi-, poly- et asexuelles, les auteurs ne reconnaissent pas que l'orientation sexuelle ne s'inscrit pas seulement dans une logique de différenciation - la norme hétérosexuelle et son contraire - mais est aussi plurielle. De plus, se baser sur des expressions de genre typiques comme dénominateur commun de l'étude - en n'incluant pas les personnes transgenres, non-binaires et intersexuées, renforce une vision binaire et cisgenre du monde.

6 Ce projet vise à interroger la nécessité d'une telle classification et le rôle que joue la photographie dans celle-ci.

7 Avec le développement de programmes de reconnaissance faciale, un nouveau rôle est assigné à la photographie sur le plan scientifique. La sensibilité du photographe est diminuée afin de se concentrer sur le sujet. Seule la "réalité » de la personne et ses caractéristiques physiques demeurent. Le but de ce travail est de ramener le débat à un niveau humain et de révéler la perception et la construction d'orientations sexuelles dans un contexte post-technologique. Une esthétique similaire aux études physiognomoniques du XIX siècle est utilisée afin de mettre en lumière la longue tradition de ces recherches pseudo-scientifiques aspirant à tirer de l'observation physiques des lois immuables sur la personnalité ou le caractère de la personne observée. J'utilise les poses et les positions de la tête analysées par l'algorithme de Kang et Kowinski dans leur étude, je cherche cependant sciemment des traces d'individualité dans chacune des sujets.

Dans le cadre de ce projet, je développe des séries de portraits composés de neuf prises de vue qui reprennent d'une part les positions du visage et de la tête analysées et reconnues par le programme de reconnaissance faciale utilisé par Wang et Kosinski dans leur étude. D'autre part, l'esthétique et le grain de l'argentique rappelle sciemment les études photographiques de physiognomonie du XIX ${ }^{\mathrm{e}}$ siècle qui visaient déjà à utiliser l'objectivité supposée de la photographie pour analyser le physique de certaines classes et/ou populations et en déduire leur caractère ou leur moralité. En reprenant les codes de cette esthétique, il est alors possible d'établir un lien historique entre les études de cette pseudo-science, élément fondateur du racisme scientifique et tombée en désuétude faute de preuve, et son renouveau scientifique actuel rendu possible par le développement de programmes de reconnaissance faciale. 
Image 3

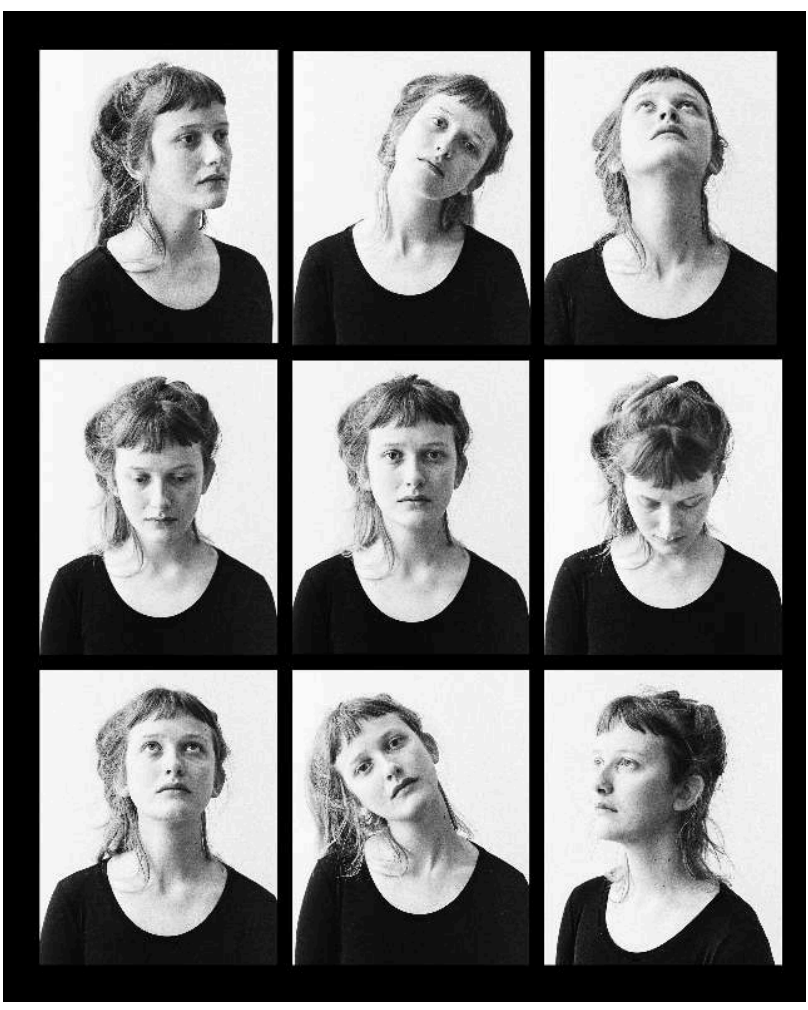

Image 4

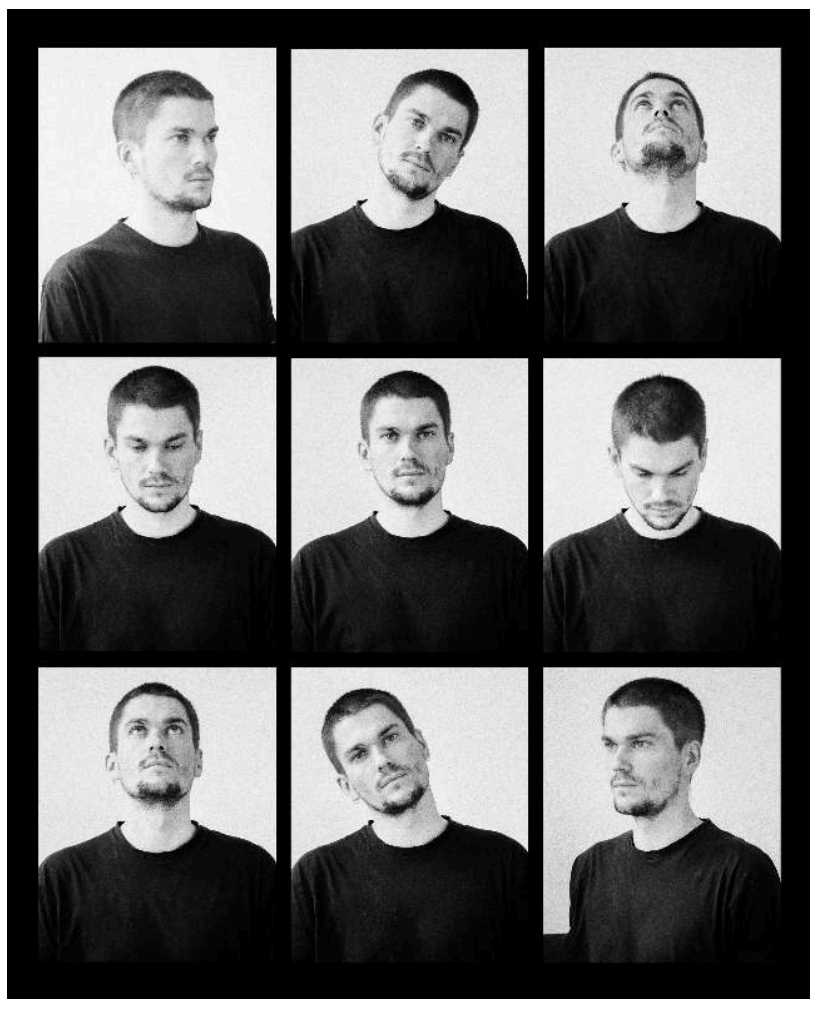


9 Un autre aspect de ce travail se traduit par une installation interactive qui se réfère à la technologie de reconnaissance faciale utilisée par Wang et Kosinski et reprend les codes utilisés lors de cette étude de façon ironique.

L'installation se compose d'un ordinateur à hauteur du public sur un socle. Il est équipé d'une webcam et diffuse en direct les images sur l'écran. D'autre part, un fond de studio photo permet de créer une « scène " sur laquelle les visiteur·euse's peuvent se mouvoir et qui symbolise également la construction et la théâtralisation de leur propre identité.

Image 5

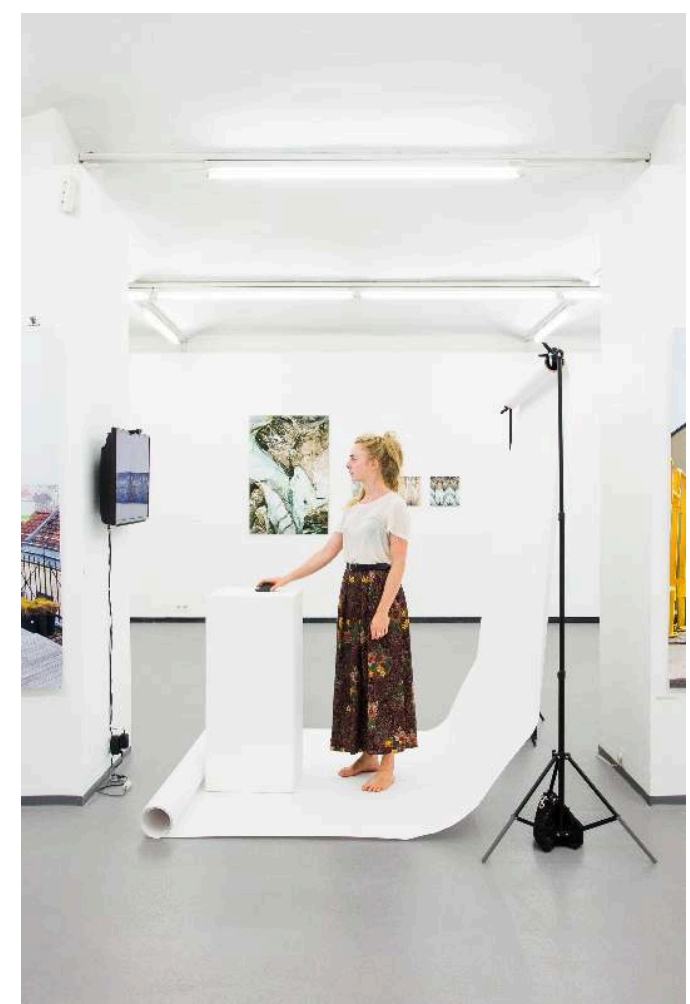




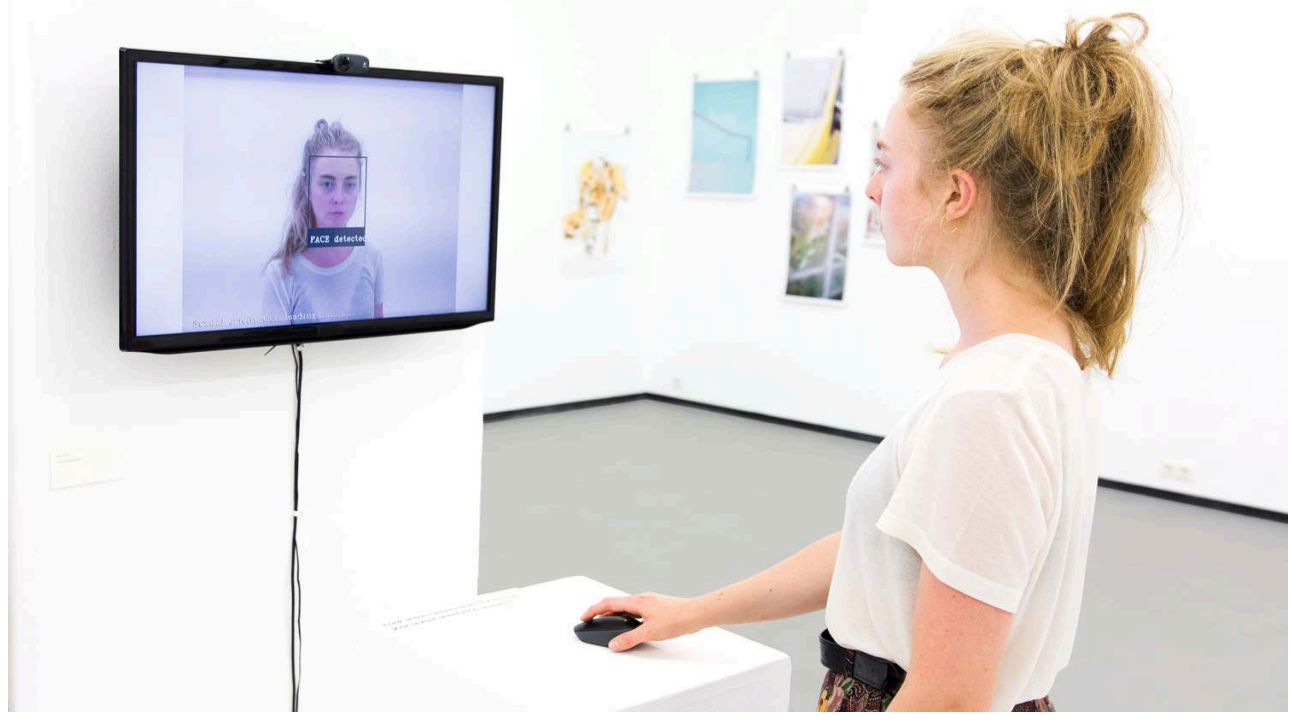

Image 7

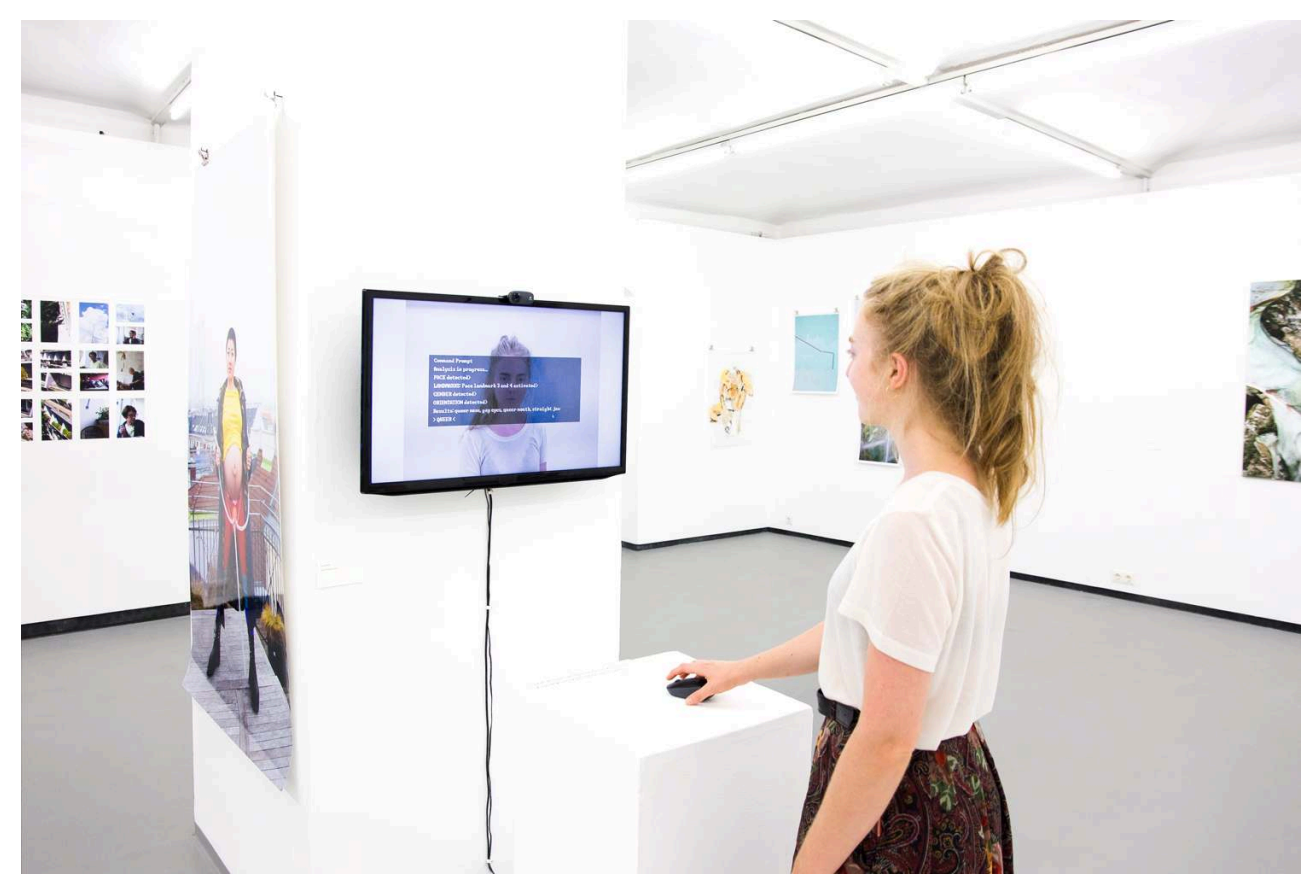

11 En déclenchant la webcam, ils activent alors l'ordinateur censé classifier et reconnaître leur orientation sexuelle. Cependant, il n'est pas question ici de conduire de réelles analyses, le programme montre des résultats arbitraires et aléatoires. Ceci permet alors une confrontation entre la perception de sa propre orientation sexuelle et la projection possible de celle-ci en tant que construction sociale dans un contexte posttechnologique.

12 De plus, afin de poser un regard critique sur la vision hétéronormative de l'étude de Standford, ces fausses analyses proposent une version queer du monde et de 
l'orientation sexuelle puisque seulement 5 résultats sont proposés de façon aléatoire (GAY; BISEXUAL, ASEXUAL; POLYSEXUAL; QUEER) et l'hétérosexualité n'en faisant pas partie.

Ce média ne peut être affiché ici. Veuillez vous reporter à l'édition en ligne http:// journals.openedition.org/glad/1737

\section{INDEX}

Thèmes: Créations

Mots-clés : intelligence artificielle, reconnaissance faciale, orientation sexuelle, photographie, interactif

Keywords : artificial intelligence, facial recognition, sexual orientation, photography, interactive

\section{AUTEUR}

\section{ÉLODIE GRETHEN}

Élodie Grethen (1988) est une artiste française vivant à Vienne. En 2016, elle publie Tokyo Stories, un livre de photographie classé parmi les 15 plus beaux livres d'Autriche 2017 et parmi les finalistes du Kassel Dummy Award 2017. En 2018, elle sort diplômée de l'école de photographie artistique Friedl Kubelka et elle expose notamment au Kunstforum Wien, Fotogalerie Wien et Camera Austria. En 2018, elle reçoit l'une des 5 "Start" bourses en photographie d'art de la chancellerie autrichienne.

Elle comprend son travail comme l'exploration des structures sociales en place dans le monde. En mettant l'accent sur la notion de genre, d'intimité et d'appartenance, elle utilise sa pratique artistique pour refléter la construction des identités individuelles et sociales et pour étudier la relation entre les individus et la société. 